\title{
Fabricación y caracterización de aleaciones porosas de Ti y Ti6Al4V producidas mediante sinterización con espaciador ${ }^{(*)}$
}

\author{
C. Tojal*, V. Amigó* y J.A. Calero**
}

\begin{abstract}
Resumen
El titanio es un material biocompatible que, además de presentar buenas propiedades a la corrosión, posee una elevada resistencia mecánica teniendo en cuenta su baja densidad. En el campo de la pulvimetalurgia, entre otras aplicaciones, este material se usa con objeto de obtener materiales porosos para aplicaciones biomédicas. Recientemente se ha investigado la aplicación de los materiales porosos en la fabricación de implantes de cadera. La razón principal está basada en la reducción de la rigidez de los implantes, lo cual minimiza los efectos del "apantallamiento de tensiones", al aproximarse su módulo elástico al del hueso. El propósito del presente trabajo, es producir materiales porosos mediante la técnica de sinterización con espaciador, usando el bicarbonato de amonio como propulsor de la formación de poros. Para la obtención de los mismos, se ha utilizado polvo de titanio de diferentes tamaños de partícula, usando diversas presiones de compactación. Antes de realizar la sinterización, se han evaluado las propiedades mecánicas de las muestras en verde, de modo que se permita su manipulación. Tras realizar la sinterización, se ha evaluado la densidad y porosidad. Igualmente, se ha valorado el efecto de estas variables en las propiedades mecánicas y el módulo elástico, obtenidos mediante el ensayo de flexión a tres puntos. La caracterización microestructural se ha realizado mediante microscopía óptica y electrónica.
\end{abstract}

\section{Fabrication and characterisation of porous Ti and Ti6Al4V produced by sintering with spacer}

\begin{abstract}
Titanium is well-known to be a biocompatible material with good corrosion properties and good strength, taking into account their low specific weight. In powder metallurgy field, titanium has been used in order to obtain porosity materials for biomedical applications. Recently, porous materials have been investigated for their use like hips implants. The principal reason is based on a reduction of stiffness implants, minimizing effects of stress shielding. The purpose of the present work is produced porous materials by space holder technique using ammonium bicarbonate like spacer. Scaffolds of titanium have been fabricated by powders of titanium with different grades of particle size and compacting pressure. Before sintering, stability of green parts has been studied by mechanical test. After sintering, porosity has been evaluated besides mechanical properties and elastic modulus by three points bending test. The microstructural characterisation is performed by optical and electron microscopy.
\end{abstract}

Keywords

Porous titanium; Stiffness; Space-holder method; Ammonium bicarbonate; Particle size; Bending test.

\section{INTRODUCCIÓN}

En el campo de la pulvimetalurgia, el titanio se usa con objeto de obtener tanto materiales densos para aplicaciones industriales ${ }^{[1 \text { y } 2]}$, como materiales porosos para aplicaciones biomédicas ${ }^{[3]}$. El titanio es muy conocido por ser un material biocompatible con buena resistencia a la corrosión ${ }^{[4]}$. Como valor añadido, tiene la ventaja de presentar una buena resistencia mecánica, en comparación con su peso, lo cual resulta apropiado para su uso como implantes óseos. La aleación de titanio más usada es la conocida Ti6Al4V: esta aleación presenta una estructura bifásica $(\alpha+\beta)$ lo cual le transfiere buenas propiedades mecánicas ${ }^{[5]}$.

Desde hace años se viene estudiando el proceso de obtención de materiales porosos desarrollados por

(·) Trabajo recibido el día 1 de febrero de 2012 y aceptado en su forma final el día 11 de junio de 2012.

Instituto de Tecnología de Materiales, Universidad Politécnica de Valencia (UPV), Camino de Vera s/n, 46022, Valencia.

E-mail:vamigo@mcm.upv.es.

** Aleaciones Metálicas Sinterizadas (AMES), Ctra. Laureà Miró, 388 -08980 Sant Feliú de Llobregat, Barcelona. E-mail: jacalero@ames.es. 
vía pulvimetalúrgica para la fabricación de implantes $^{[6]}$. El propósito del uso de estos materiales se debe al valor de la propiedad de rigidez que presentan los mismos; una gran diferencia entre la rigidez del hueso y del implante metálico, tiene como consecuencia una diferente distribución de tensiones, "apantallamiento de tensiones", lo cual genera una pérdida de masa ósea en el tejido óseo circundante que suele acabar en fractura ósea ${ }^{[7]}$. Este problema se puede evitar usando implantes con una menor rigidez, más parecida a la del hueso. Los materiales porosos resultan unos candidatos ideales ya que su rigidez es menor que la de los materiales densos. Como valor añadido, con la superficie rugosa de los materiales porosos se facilita la osteointegración, aportando una mayor superficie de fijación y una mejor transmisión de esfuerzos entre el implante y el hueso ${ }^{[8]}$, lo que resulta en una unión biológica entre el implante y el tejido vivo $^{[9]}$.

Para obtener espumas de titanio por pulvimetalurgia se utilizan favorablemente polvos de hidruro de titanio $^{[10]}$. Una técnica empleada en los últimos años es la técnica del espaciador ${ }^{[8}$ y 11$]$. Esta técnica consiste en sinterizar un material que previamente se ha mezclado con otro material (llamado espaciador), el cual se eliminará en un paso previo a la sinterización, dejando huecos en su lugar. De esta forma, se pueden obtener materiales de elevada porosidad. El bicarbonato de amonio es un espaciador que puede ser eliminado fácilmente por descomposición térmica ${ }^{[12]}$.

La fabricación de polvos de titanio no es fácil debido a la alta reactividad que presenta este material ${ }^{[13]}$, y por ello se recurre en muchos casos a técnicas de hidruración-deshidruración (HDH) como método para obtener polvo de titanio ${ }^{[14]}$, consistente en producir hidruro de titanio y posteriormente fracturar el material para obtener polvo de hidruro de titanio. En este proceso, el último paso es la reducción del material para obtener polvo de titanio. Por lo tanto, la reactividad del material durante el proceso se inhibe y la rugosidad que adquieren los polvos permite una buena compactación uniaxial ${ }^{[15]}$.

El propósito del presente trabajo es producir materiales porosos basados en polvo de titanio sinterizado mediante la técnica del espaciador, usando bicarbonato de amonio como formador de porosidad. La razón del uso de esta técnica se basa en la producción de materiales con formas casi acabadas y de porosidad controlada, lo que permite a su vez, controlar su rigidez. Tras la obtención de los compactos con polvos de diferente tamaño de partícula a distintas presiones de compactación, se estudiará su estabilidad mediante ensayos de flexión a tres puntos. Después de la sinterización y tras evaluar su porosidad final, se realizarán nuevamente ensayos de flexión a tres puntos para obtener tanto sus propiedades mecánicas, como el módulo de elasticidad en función del tamaño de partícula utilizado. La caracterización microestructural de las aleaciones se ha llevado a cabo por microscopía óptica y electrónica.

\section{MATERIALES Y PROCEDIMIENTO EXPERIMENTAL}

Los polvos de titanio y la aleación Ti6A14V fueron suministrados por Se-Jong Materials (Korea del Sur). El proceso de fabricación de los polvos fue por hidruración-deshidruración $(\mathrm{HDH})$, siendo partículas de forma irregular con tamaño menor de $45 \mu \mathrm{m}$ (dato aportado por el fabricante). La composición de cada polvo se observa en la tabla I.

Tabla I. Composición química de los polvos de titanio y de Ti6Al4V

Table I: Chemical composition of titanium and Ti6Al4V powder

$\mathrm{Ti}$

Composición (\% peso)

\begin{tabular}{cccccccc}
\hline Ti & Mg & Si & Mn & Fe & O & N & H \\
\hline 99,7 & 0,01 & 0,09 & 0,01 & 0,03 & 0,36 & 0,01 & 0,01 \\
\hline \multicolumn{8}{c}{$\begin{array}{c}\text { Ti6Al4V } \\
\text { Composición (\% }\end{array}$} \\
\hline Ti & Al & V & Fe & Zr & O & N & H \\
\hline 88,8 & 6,62 & 4,55 & 0,02 & 0,03 & 0,55 & 0,5 & 0,3 \\
\hline
\end{tabular}

Ambos materiales han sido tamizados con objeto de obtener dos fracciones de polvo, por encima y por debajo de $25 \mu \mathrm{m}$. En resumen, se ha trabajado con diferentes fracciones dando lugar a tres distribuciones granulométricas: la primera sin tamizar; la segunda, tamizada por debajo de $25 \mu \mathrm{m}$ y la tercera, tamizada por encima de $25 \mu \mathrm{m}$. Estas tres distribuciones granulométricas se pueden observar en la figura 1 .

Hay que hacer notar que se observan distribuciones de tamaño mayores a la esperada para el polvo tamizado por debajo de $25 \mu \mathrm{m}$, debido a la forma irregular de las partículas por el propio proceso de fabricación, que posteriormente el equipo de determinación granulométrica (Matersizer 2000 Malver Instruments) evalúa. 


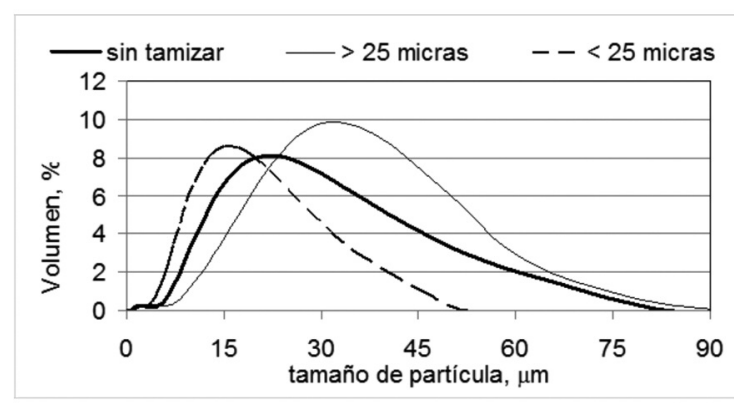

a)

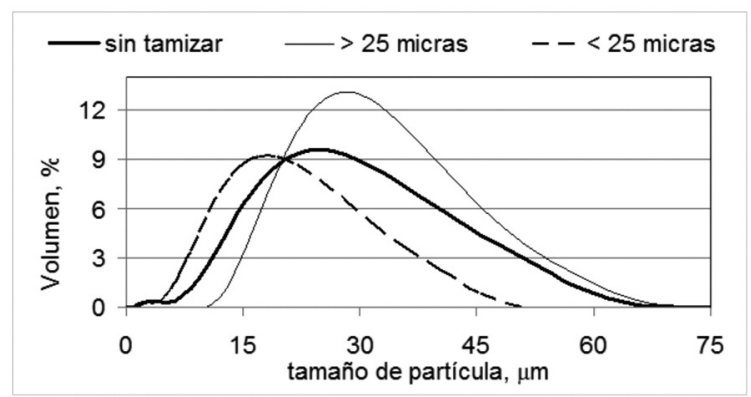

b)

Figura 1. Análisis del tamaño de partícula de los materiales investigados: a) Ti, b) Ti6Al4V.

\section{Figure 1. Sieve analysis of investigated materials: a) Ti, b) Ti6Al4V.}

Como material espaciador se ha usado bicarbonato de amonio, el cual ha sido suministrado por Alfa Aesar. El espaciador ha sido tamizado entre 425 y $600 \mu \mathrm{m}$ con objeto de obtener las mejores propiedades, tal como se indicó en trabajos anteriores ${ }^{[16}$ y 17].

La mezcla de materiales con el espaciador se llevó a cabo en un único paso, con una mezcladora (Inversina $2 \mathrm{~L}$ Bioengineering) a la velocidad de $38 \mathrm{rpm}$, en porcentajes de 60,70 y $80 \%$ en volumen de espaciador. Se han investigado los materiales sin espaciador añadido, para utilizarlos como referencia y poder de esta forma comparar y entender mejor los resultados.

El segundo paso es la compactación uniaxial. Las mezclas de polvos fueron compactadas entre 200 y $500 \mathrm{MPa}$, mediante el equipo universal de ensayos Instron modelo 8000, evaluando la curva de compactación para cada mezcla, en una matriz flotante diseñada con objeto de producir probetas adecuadas para realizar el ensayo de flexión, de dimensiones $12 \times 30 \mathrm{~mm}^{2}$, obteniendo una altura de los compactos en torno a $6,5 \mathrm{~mm}$.

El bicarbonato de amonio fue eliminado en una estufa (Carbolite $500^{\circ} \mathrm{C}$ ). La temperatura del horno se mantuvo entre 353 y $393 \mathrm{~K}$, con un tiempo de exposición de hasta $24 \mathrm{~h}$.

La sinterización se realizó en un horno de alta temperatura Carbolite modelo HVT 15/75/450 en condiciones de alto vacío, necesario durante la sinterización del titanio para evitar la reactividad de este elemento ${ }^{[18]}$. El proceso de sinterización se ha realizado a $1523 \mathrm{~K}$ durante $2 \mathrm{~h}$ usando velocidades de calentamiento y enfriamiento entre 10 y $15 \mathrm{~K} \cdot \mathrm{min}^{-1}$ y manteniendo un vacío durante todo el proceso de al menos $10^{-4} \mathrm{~atm}$.

La densidad de las muestras se ha evaluado por el método de Arquímedes de acuerdo con la norma ASTM C 373-88. Con la densidad obtenida, se halló la porosidad abierta y cerrada de las muestras.
El ensayo de resistencia mecánica ha tenido lugar con una prensa Instron modelo 4204. Con objeto de evaluar la resistencia de las muestras en verde, se sometieron dichas muestras al ensayo de flexión a tres puntos, de acuerdo con la norma ISO 3325, usando una velocidad de $0,5 \mathrm{~mm} \mathrm{~min}^{-1}$ y una célula de carga de $1 \mathrm{kN}$. Después de la sinterización, las muestras fueron sometidas al mismo ensayo mecánico, con objeto de evaluar la resistencia máxima y el módulo a flexión, el cual se comparó con el módulo obtenido para las aleaciones de forja ensayadas en las mismas condiciones (21 GPa para titanio y 29 GPa para Ti6Al4V). Para ensayar las muestras sinterizadas mediante el ensayo de flexión a tres puntos, nuevamente se utilizó la prensa Instron modelo 4204 con la misma velocidad de $0,5 \mathrm{~mm} \mathrm{~min}^{-1}$ aunque la célula de carga fue de $5 \mathrm{kN}$ para materiales porosos y $50 \mathrm{kN}$ para los materiales densos de forja.

Tras el ensayo de flexión se realiza un estudio fractográfico mediante microscopía electrónica de barrido con equipo JEOL JSM-6300. Para el análisis microestructural se realiza la preparación metalográfica de las muestras .El ataque químico se realiza con el reactivo de Kroll ( $3 \mathrm{ml} \mathrm{HF}, 6 \mathrm{ml} \mathrm{HNO}$ y $100 \mathrm{ml} \mathrm{H}_{2} \mathrm{O}$ ), revelándose la microestructura y evaluando el tamaño de grano. Las muestras se observan mediante el microscopio óptico Nikon Microphot FX y el microscopio electrónico JEOL JSM-6300.

\section{RESULTADOS Y DISCUSIÓN}

\subsection{Eliminación del espaciador}

La correcta eliminación del espaciador resulta un paso fundamental en el proceso de obtención de materiales porosos por la técnica de sinterización con espaciador. Además, el espaciador utilizado, no debe 
afectar a la composición química del material durante todo el proceso de fabricación. En este estudio se ha usado como espaciador el bicarbonato de amonio, el cual no reacciona con el titanio durante el proceso ${ }^{[12}$ y 19]. El bicarbonato de amonio se elimina fácilmente por aporte de calor, trabajando a temperaturas superiores a su temperatura de descomposición térmica (308 K). Un posible problema surge al intentar conseguir la total eliminación de este compuesto sin crear defectos mecánicos por la expansión que tiene en la formación de amoniaco gas. En la figura 2 se ha representado la pérdida de peso, durante la exposición en la estufa a diferentes temperaturas, para muestras de titanio fabricadas con un $60 \%$ de espaciador.

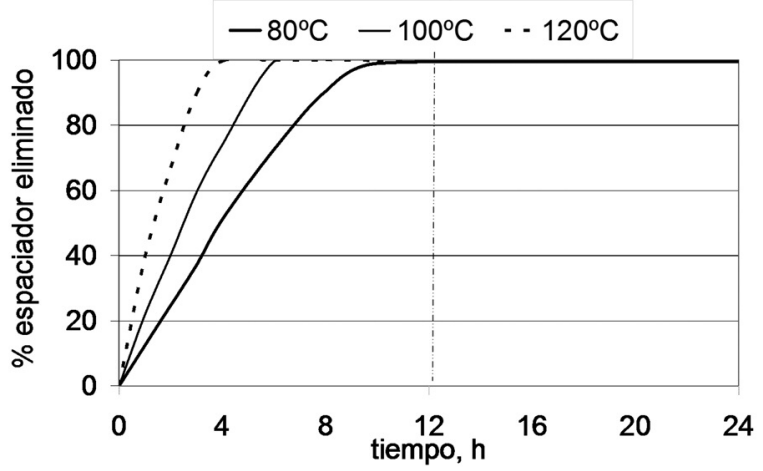

Figura 2. Cantidad de bicarbonato de amonio eliminado en horno en función del tiempo de exposición para muestras preparadas con un $60 \%$ de espaciador.

Figure 2. Quantity of ammonium bicarbonate removed in furnace related to time of exposition for samples manufactured with $60 \%$ spacer.
El tiempo necesario para eliminar el espaciador es mayor cuanto menor es la temperatura del horno, pero en todos los casos, $12 \mathrm{~h}$ resulta un tiempo adecuado para asegurar la eliminación total. Aumentando la temperatura del horno, podría aumentar la probabilidad de dañar los compactos. Sin embargo, no se han observado daños aparentes en muestras tratadas a $373 \mathrm{~K}$, y a esta temperatura, se garantiza la total eliminación del bicarbonato y la humedad. Es por esta razón que las muestras compactadas se introdujeron en la estufa a $373 \mathrm{~K}$ durante $12 \mathrm{~h}$, con objeto de crear la estructura porosa antes de la sinterización.

\subsection{Estabilidad de los compactos en verde}

Resulta relevante evaluar la estabilidad de los compactos en verde, debido a que la introducción del espaciador provoca una manipulación menos estable. La presión de compactación tiene una notable influencia en la resistencia de los compactos en verde. Los resultados de resistencia de las muestras en verde pueden observarse en la figura 3 .

Aunque la diferencia de resistencias obtenidas no es excesivamente alta, los compactos fabricados con polvos tamizados tienen una menor resistencia que el resto. La resistencia mayor se obtiene para compactos fabricados a partir de polvos que no han sido tamizados. La densidad de empaquetamiento es la razón por la cual se explican estos resultados ${ }^{[20]}$ : una mayor densidad de empaquetamiento se obtiene cuando hay partículas pequeñas llenando los inters-

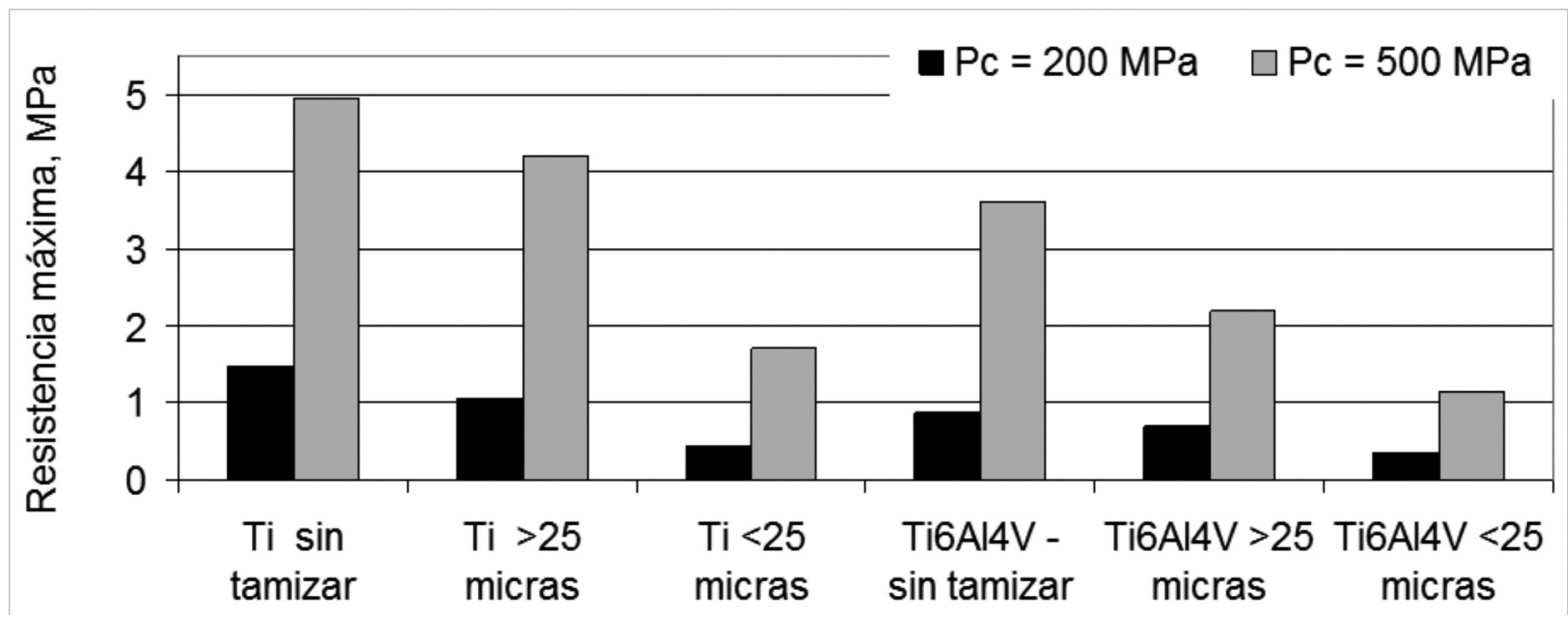

Figura 3. Resistencia máxima a flexión de las muestras en verde fabricadas con un $60 \%$ de espaciador.

Figure 3. Maximum bending resistance for green samples manufactured with $60 \%$ spacer. 
ticios que dejan partículas de mayor tamaño. Por lo tanto, una distribución más amplia de tamaños de partículas aumenta la densidad de empaquetamiento y durante el proceso de compactación se producen más contactos mecánicos, y, como consecuencia, se aporta una mayor resistencia mecánica a los compactos. No obstante es importante señalar que debe tenerse en cuenta la menor capacidad de rellenar los espacios entre las partículas de bicarbonato cuando aumenta su proporción.

\subsection{Sinterabilidad y porosidad final}

Los resultados de la densidad en verde de los compactos se muestran en la figura 4 .

Se ha encontrado que las series de polvo base titanio presentan una tendencia similar a las series de polvo base Ti6Al4V. Esta variable está más influenciada por el tipo de material y la cantidad de espaciador añadida, que por el tamaño de partícula usado para el material base. Los cambios de densidad son de un $5 \%$ entre usar la presión de compactación de $300 \mathrm{MPa}$ y la de $500 \mathrm{MPa}$. La densidad en verde se reduce en aproximadamente un $18 \%$ cuando la can- tidad de espaciador utilizada para la fabricación aumenta de un $60 \%$ a un $80 \%$.

Las propiedades de los materiales obtenidos dependen de la porosidad, la microestructura y la calidad de los cuellos de la matriz metálica. En este caso, sobre las propiedades finales, la porosidad es la variable con mayor influencia para el diseño de materiales adecuados a aplicaciones específicas. Resulta interesante que la porosidad pueda ser controlada, ya que de esta forma cabe la posibilidad de obtener biomateriales con tamaños de poro adecuados para mejorar el crecimiento celular y la fijación de los implantes. La presión de compactación usada genera diversos cambios pero sin una tendencia clara, tal como puede observarse en la figura 5.

Esto se debe a que los mecanismos de contracción durante la sinterización, están dominados no sólo por la densidad de los compactos después de la compactación, sino también por la porosidad creada después de la eliminación del espaciador. La porosidad final está íntimamente relacionada con la cantidad de espaciador usada durante la fabricación de los compactos $^{[21]}$. La porosidad es mayoritariamente abierta en todos los casos, lo cual permite un mejor crecimiento celular.
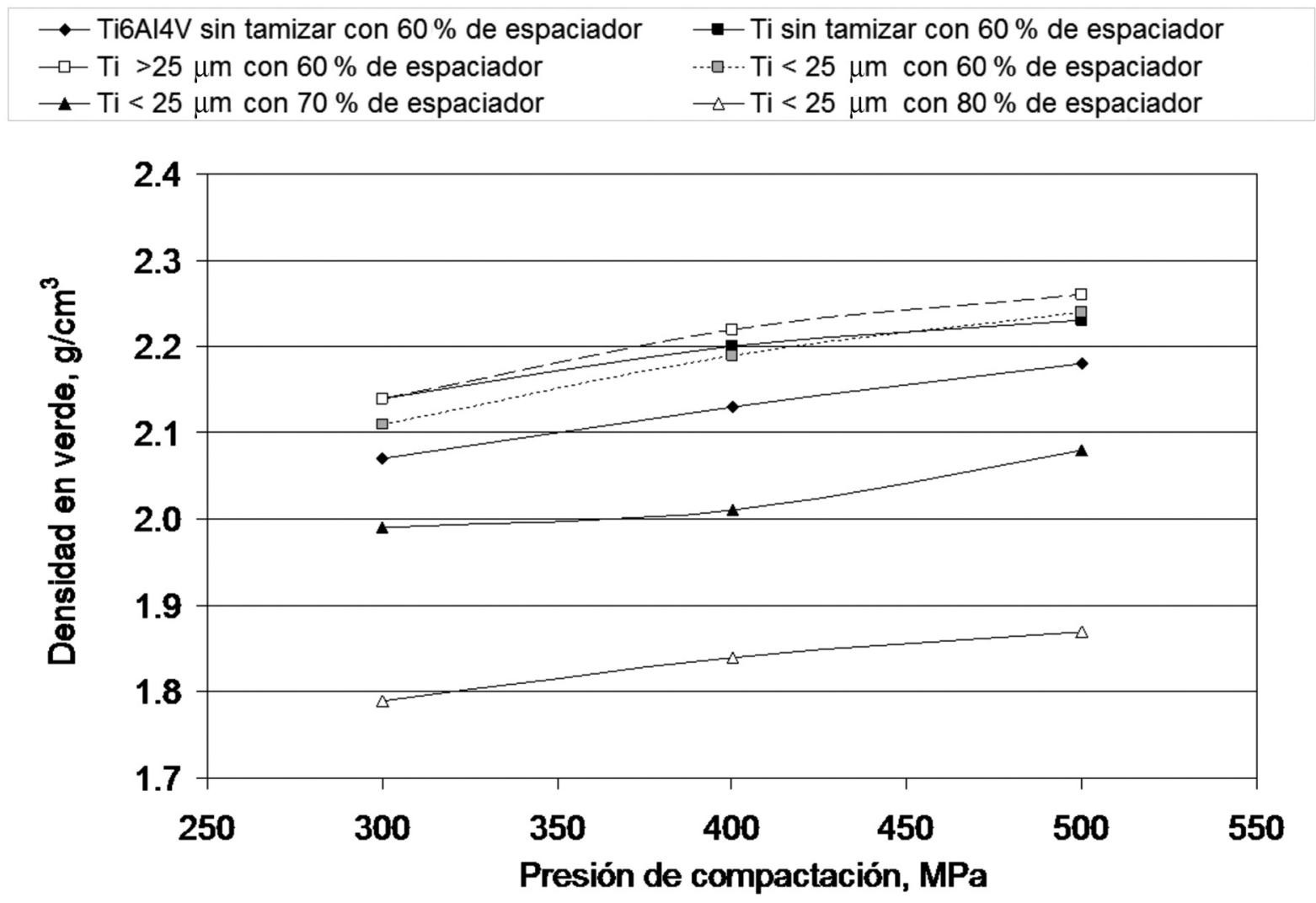

Figura 4. Densidad en verde de las muestras.

Figure 4. Green density of samples. 


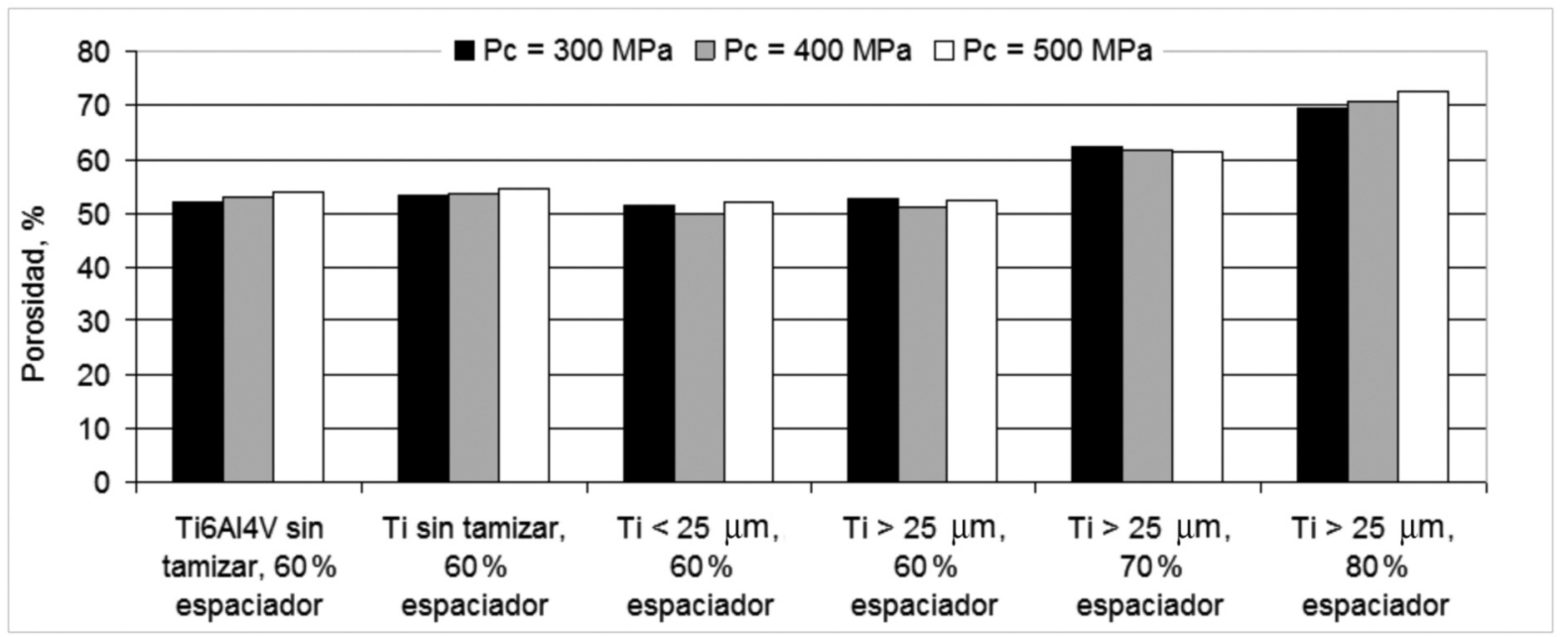

Figura 5. Porosidad de las muestras.

Figure 5. Porosity of samples.

\subsection{Propiedades mecánicas}

Como se ha mostrado la presión de compactación no tiene más que una pequeña influencia en la porosidad final de los materiales estudiados, por lo que no se observan importantes variaciones entre las propiedades mecánicas, al considerar esta variable. Los resultados de los ensayos de flexión para muestras compactadas a una presión de compactación intermedia, de $400 \mathrm{MPa}$, se muestran en la figura 6 .

Los valores de resistencia máxima para el titanio y Ti6Al4V (Fig. 6 a)) son muy similares independientemente del tamaño de partícula usado. Por otro lado, los valores del módulo tienen una clara tendencia a disminuir ligeramente cuando el tamaño de partícula usado es mayor de $25 \mu \mathrm{m}$. Se ha encontrado que las propiedades mecánicas dependen de la porosidad, disminuyendo cuando la porosidad aumenta ${ }^{[22]}$. Se ha observado que el valor de la resistencia está altamente relacionado con el grado de porosidad de las muestras, más que con el tamaño de partícula utilizado. Es por ello que, como dato de referencia, se indica una caída del valor de la resistencia en torno a $150 \mathrm{MPa}$ para muestras fabricadas con un $80 \%$ de espaciador. Por lo tanto, sobre la propiedad de rigidez, se ha encontrado que los resultados dependen en cierta medida del tamaño de partícula usado, como puede observarse en la figura 6 b); el valor del módulo es menor para muestras con tamaño de partícula superior a $25 \mu \mathrm{m}$, y mayor para muestras donde no se tamizó el polvo base. Como resulta lógico, el módulo relativo al obtenido para los correspondientes mate- riales densos, es menor cuanto mayor es la cantidad de espaciador usado para su fabricación y la porosidad creada (Fig. 6 c)).

\subsection{Microestructura}

Mediante microscopía óptica se han obtenido micrografías de muestras de titanio poroso preparadas con diferentes tamaños de partícula, las cuales se representan en la figura 7 .

La influencia del tamaño de partícula es evidente en la morfología del poro de las muestras: hay una gran existencia de porosidad abierta, debido a la presencia de espaciador, pero la porosidad cerrada es más evidente en muestras con mayor tamaño de partícula. La contracción durante la sinterización ocurre cuando el mecanismo de transporte de masa durante dicha sinterización tiene lugar por volumen, cuando el transporte de masa tiene lugar desde los contactos de las partículas a los cuellos de unión ${ }^{[23]}$. La teoría de Herring ${ }^{[20]}$ indica que partículas con mayor tamaño, necesitan mayor tiempo de sinterización para obtener similares resultados de porosidad después de la sinterización. El área superficial es un término que mejora el proceso de sinterización y a menor tamaño de partícula, mayor área superficial. Esta es la razón por la que se da una peor sinterabilidad y por lo tanto mayor porosidad en materiales sinterizados con mayores tamaños de partícula. El valor de la porosidad cerrada dentro de la estructura no resulta fácilmente detectable debido al alto nivel de porosidad. Es posible que pequeños cambios en las propiedades mecánicas, como la rigidez del material, estén rela- 


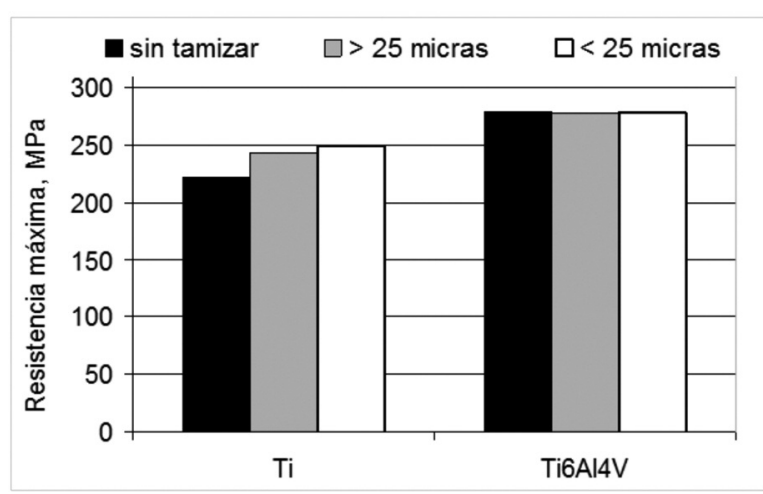

a)

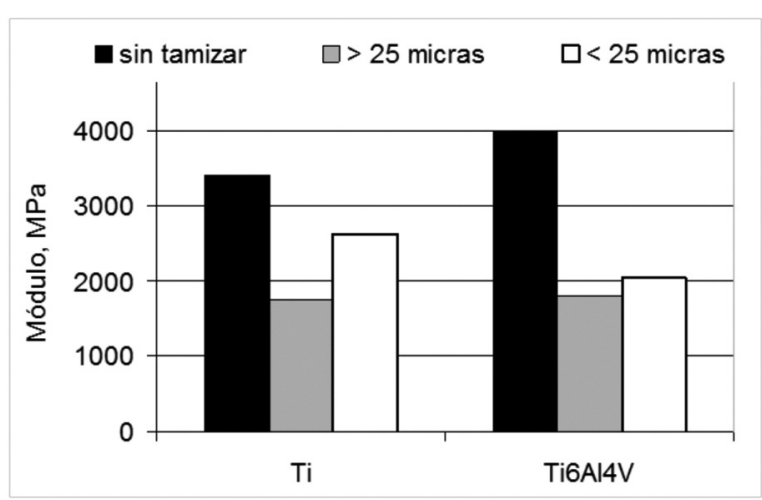

b)

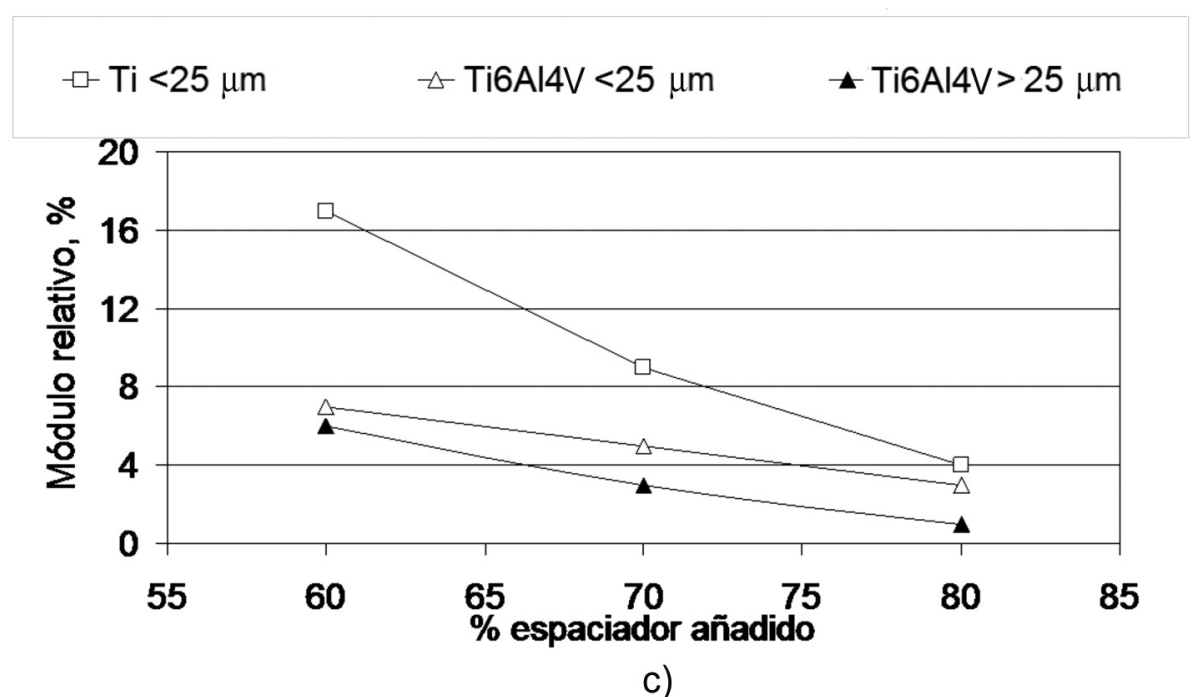

c)

Figura 6. Resultados tras ensayos de flexión para muestras porosas de titanio y Ti6Al4V (tamaño de grano indicado) compactadas a $400 \mathrm{MPa}$ : a) Resistencia máxima para muestras fabricadas con un $60 \%$ de espaciador, b) Módulo a flexión para muestras fabricadas con un $60 \%$ de espaciador, c) Módulo relativo a los correspondientes materiales de colada frente al $\%$ de espaciador.

Figure 6. Bending test results for titanium and Ti6Al4V (grain size indicated) porous samples compacted at $400 \mathrm{MPa}$ : a) Maximum resistance for samples manufactured with $60 \%$ spacer, b) Bending test modulus for samples manufactured with $60 \%$ spacer, c) Relative modulus to cast alloys versus \% spacer.

cionados con la existencia de este tipo de porosidad, aunque el efecto quedaría enmascarado en algunos casos por el alto nivel de porosidad.

Resulta evidente la obtención de un incremento de la porosidad en muestras fabricadas con mayor cantidad de espaciador ${ }^{[21]}$. Las micrografías de la figura 8 son un ejemplo del aspecto que presentan las muestras con diferentes niveles de porosidad. Se observa que la forma del poro depende de la forma original de la partícula de espaciador.

El bicarbonato de amonio es un material relativamente blando que sufre deformación durante la compactación y la forma del poro resulta alargada en la dirección de compactación. Las partículas de espaciador se han tamizado entre 425 y $600 \mu \mathrm{m}$, por lo que se obtienen grandes poros de un tamaño medio cercano a $500 \mu \mathrm{m}$ con diferentes dimensiones debido a la forma alargada que adquieren los poros por la deformación durante la compactación. Los valores del tamaño de poro son mayores para muestras con mayor cantidad de espaciador añadida durante la fabricación, debido a la conexión entre partículas de espaciador. 


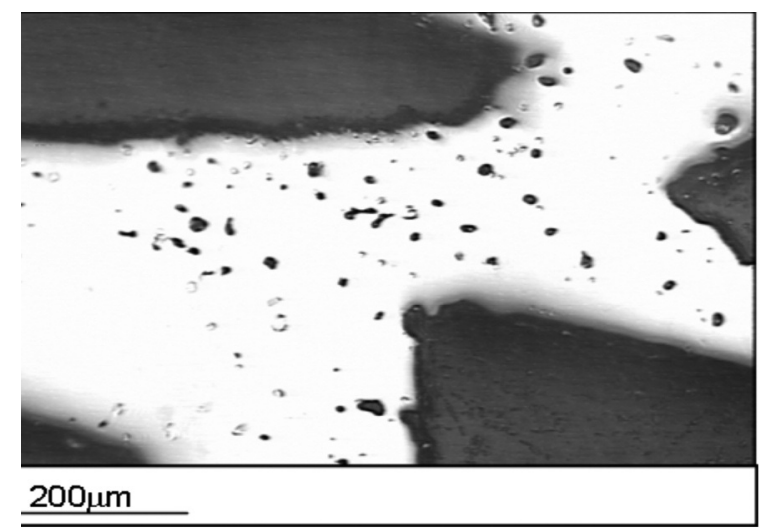

a)

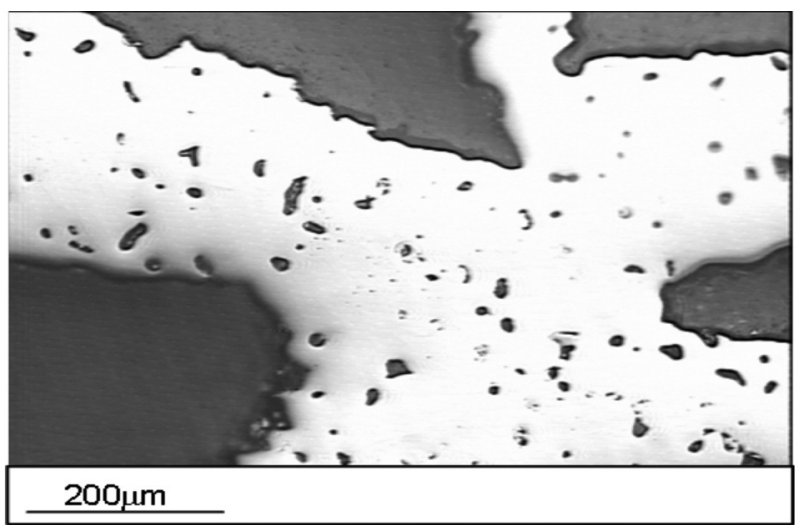

b)

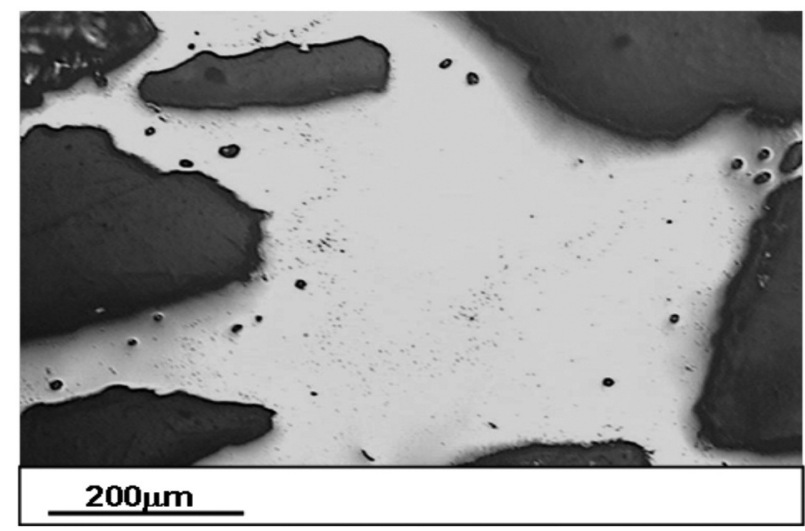

c)

Figura 7. Micrografías obtenidas por microscopía óptica de muestras porosas de titanio con diferentes tamaños de grano: a) sin tamizar, b) mayor de $25 \mu \mathrm{m}, \mathrm{c}$ ) menor de $25 \mu \mathrm{m}$.

Figure 7. Micrographs obtained by optical microscopy of porous titanium samples with different grain size: a) without sieved, b) plus $25 \mu \mathrm{m}$, c) minus $25 \mu \mathrm{m}$.

Respecto a la microestructura de las muestras, se ha observado que la presencia de porosidad cerrada tiene una influencia negativa en el propio crecimiento de grano. Si el poro se localiza en el límite de grano ${ }^{[24]}$ se inhibe el crecimiento de dicho grano. Por esta razón, para muestras preparadas con partículas con tamaños menores de $25 \mu \mathrm{m}$, se ha observado la presencia de una menor porosidad cerrada, y por lo general presentan un mayor tamaño de grano. Esto se puede observar en las micrografías de la figura 9, donde se aprecian poros cerrados colocados preferentemente en los límites de grano, previniendo el crecimiento del mismo.

Como la aleación Ti6Al4V presenta granos más finos por su estructura bifásica $(\alpha+\beta)$, la porosidad cerrada no afecta excesivamente en el crecimiento de grano. Por lo tanto, el tamaño de grano afectaría a las propiedades finales de los materiales, pero los cambios no son evidentes debido a la elevada existencia de porosidad abierta que presentan las muestras.

\subsection{Fractografías}

La típica fractura del titanio denso es dúctil ${ }^{[25]}$, pero un alto nivel de porosidad induce a una mayor fragilidad para el titanio poroso. Esto es debido a la existencia de menos puntos de contacto en proporción con el área superficial. La superficie de fractura de todas las muestras no presenta una alta deformación, como puede observarse en la fractografía de la figura $10 \mathrm{a}$ ).

Todas las muestras presentan resultados similares en este sentido, independientemente del material y del tamaño de grano usado. Existen pequeñas zonas de fractura aparentemente frágil (Fig. 10 b)) que son 


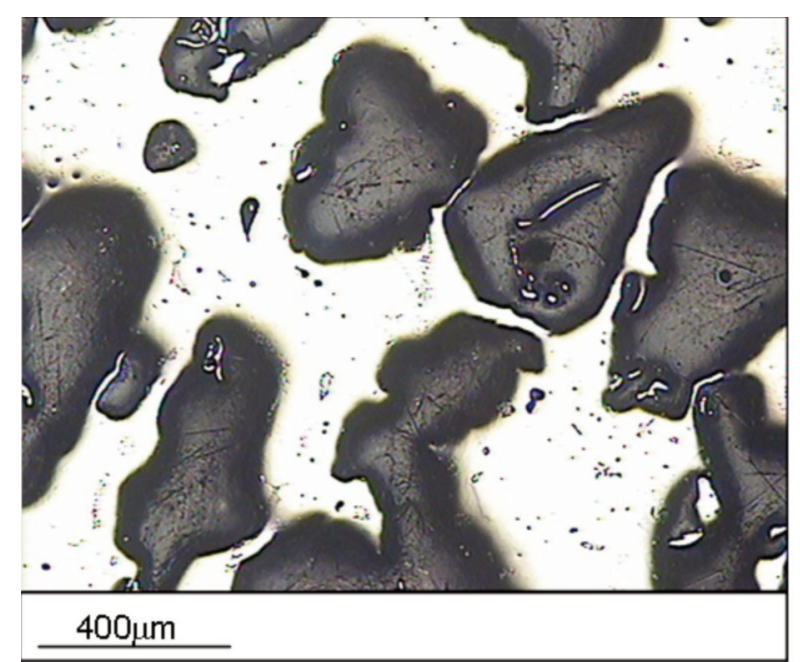

a)

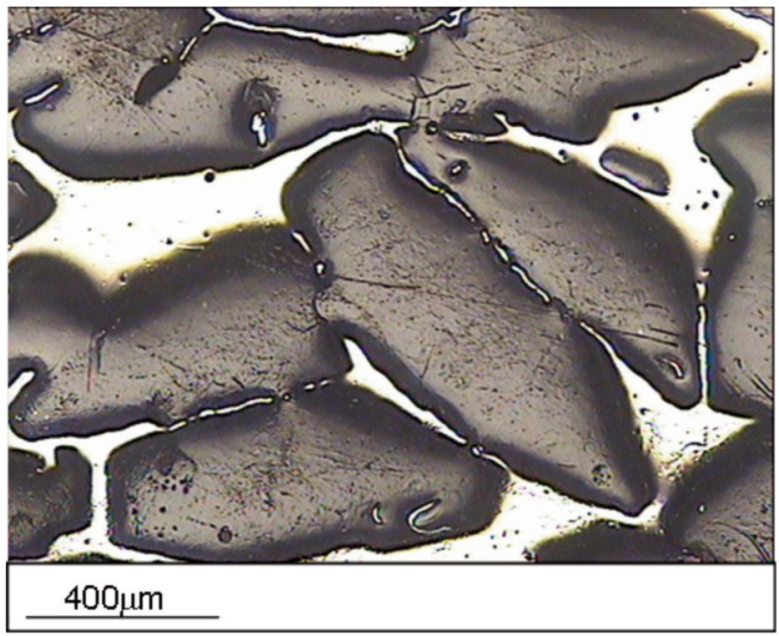

b)

Figura 8. Micrografías obtenidas por microscopía óptica para muestras porosas de Ti6Al4V (menor de $25 \mu \mathrm{m}$ ) fabricadas con: a) un $60 \%$ de espaciador, b) un $80 \%$ de espaciador.

Figure 8. Micrographs obtained by optical microscopy of Ti6Al4V (minus than $25 \mu \mathrm{m}$ ) porous samples manufactured with: a) $60 \%$ spacer, b) $80 \%$ spacer.

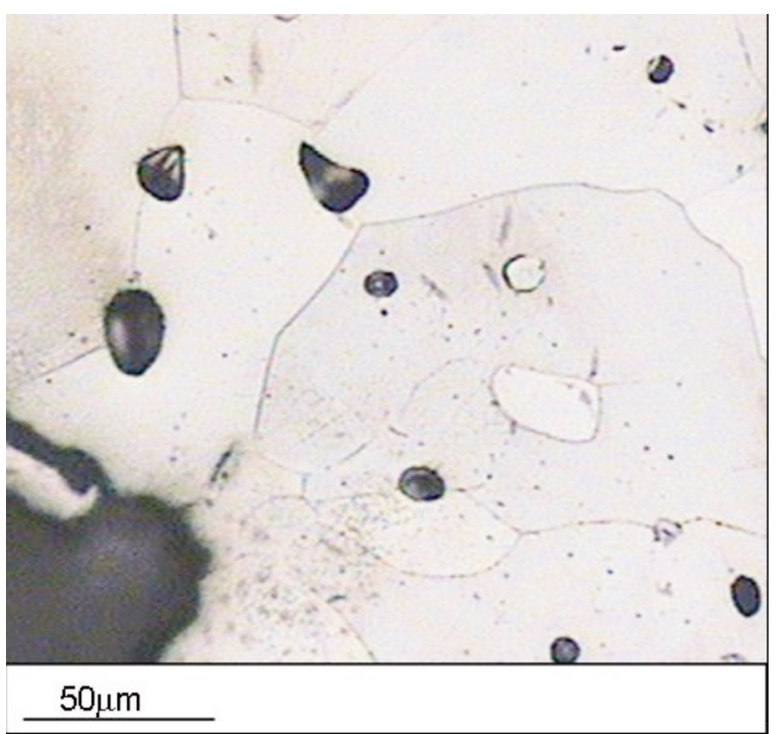

a)

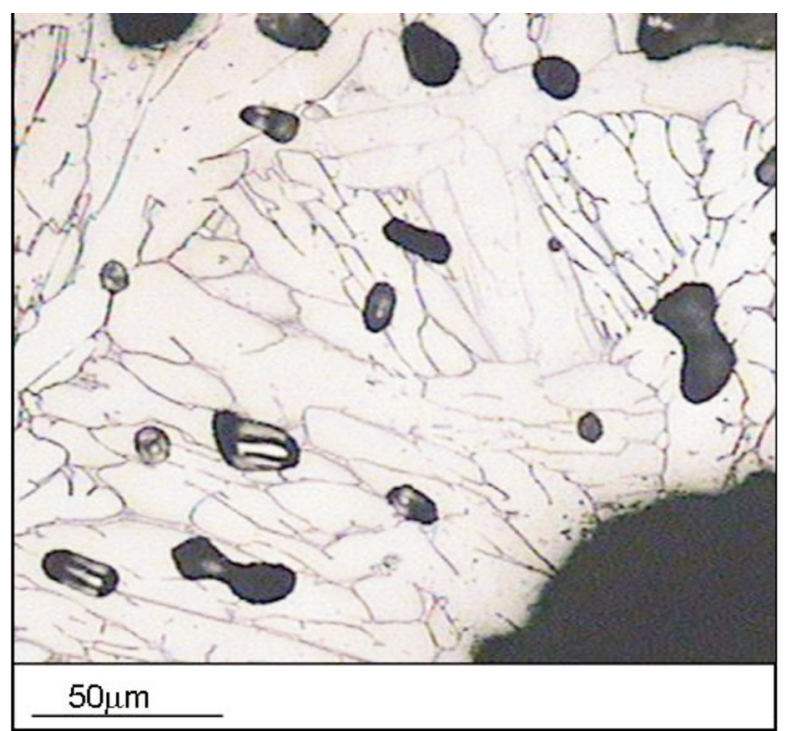

b)

Figura 9. Micrografías de detalles microestructurales obtenidas por microscopía electrónica de aleaciones porosas de muestras de titanio (mayor de $25 \mu \mathrm{m}$ ): a) Ti, b) Ti6Al4V.

Figure 9. Micrographs of microstructure details obtained by electronic microscopy of porous titanium samples (plus $25 \mu \mathrm{m}$ ): a) Ti, b) Ti6Al4V.

más difíciles de localizar a medida que se ha aumentado la cantidad de espaciador y por lo tanto el nivel de porosidad. Por otro lado, se observa que la super- ficie interna del titanio presenta una alta rugosidad, lo cual puede ayudar a una mejor osteointegración de la prótesis. 


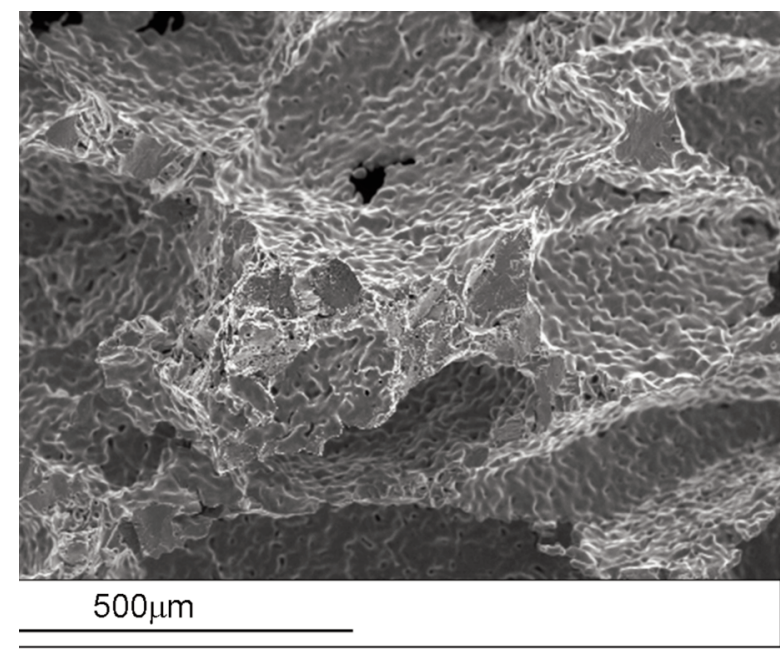

a)

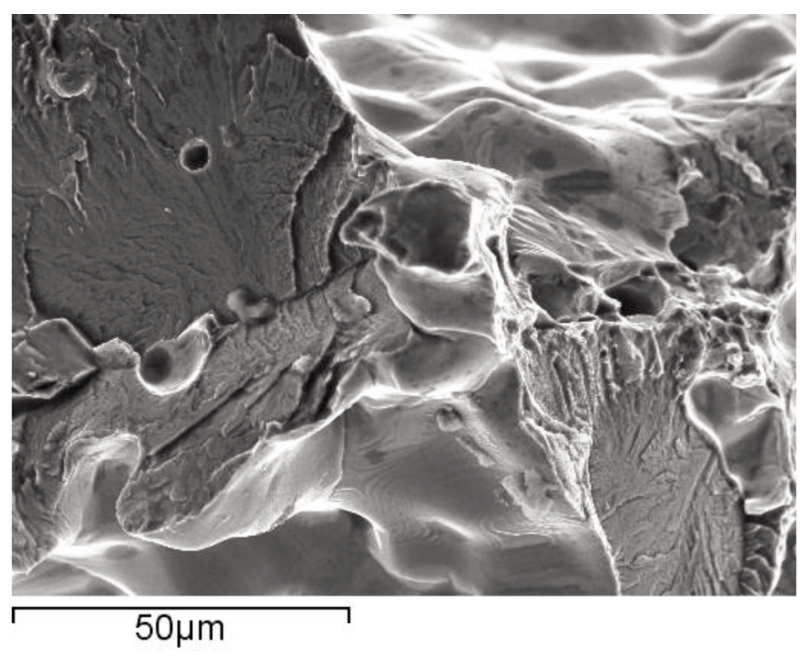

b)

Figura 10. Superficies de fractura obtenidas por microscopía electrónica de aleaciones porosas de titanio (sin tamizar) fabricadas con un $60 \%$ de espaciador: a) vista general, b) detalle de la fractura.

\section{Figure 10. Fracture surface obtained by electronic microscopy of titanium (without sieved) porous samples manufactured with $60 \%$ spacer: a) general view, b) detail of the fracture.}

\section{CONCLUSIONES}

- Se han fabricado con éxito, materiales porosos de titanio y de la aleación Ti6Al4V con diferentes grados de porosidad mediante pulvimetalurgia, usando bicarbonato de amonio como espaciador. Se han usado diferentes distribuciones granulométricas del polvo base de titanio para producir los materiales. El paso de eliminación del espaciador se ha descrito y analizado con objeto de dar a conocer una manera simple y eficaz de realizar este proceso.

- La distribución granulométrica del polvo base de titanio utilizado presenta una notable influencia sobre las propiedades de resistencia de compactos en verde, pues con una mayor distribución de partículas se obtienen compactos en verde con mejor resistencia mecánica. Sin embargo, esta variable prácticamente no afecta a la porosidad final de las muestras después de la sinterización.

- La porosidad de las muestras sinterizadas, en todos los casos, es superior al $50 \%$ y mayoritariamente abierta, dependiendo fuertemente de la cantidad de espaciador utilizado, con valores cercanos a la cantidad de espaciador introducido.

- Es, precisamente, la porosidad la variable que mayor influencia presenta en las propiedades mecánicas finales. Sin embargo la porosidad cerrada de los materiales sinterizados, como resultado de la sinterización de las paredes de la aleación, resulta afectada por el tamaño de partícula utilizada, hecho que tiene una ligera influencia en el crecimiento de grano del material de suministro. La rigidez puede resultar afectada por la modificación de esta porosidad cerrada, tal como se indica en este trabajo.

- Esta investigación muestra un proceso para fabricar materiales porosos, el cual resulta un proceso sencillo donde se puede controlar la porosidad final y propiedades mecánicas de los materiales, desarrollando de esta forma nuevas vías de producción de biomateriales para su uso como implantes óseos.

\section{Agradecimientos}

Los autores agradecen la financiación obtenida por el "Ministerio de Ciencia y Tecnología" (proyecto PET2008_0158_02).

\section{REFERENCIAS}

[1] I. Montealegre-Meléndez, E. Neubauer y H. Danninger, Powder Metall. 52 (2009) 322-328.

[2] E. Benavente-Martínez, F. Devesa y V. Amigó, Rev. Metal. Madrid 46 (№ extra) (2010) 19-25.

[3] M. Eriksson, M. Andersson, E. Adolfsson y E. Carlstrom, Powder Metall. 49 (2006) 70-77. 
[4] H. Schiefer, M. Bram, H.P. Buchkremer y D. Stover, J. Mater. Sci. Mater. Med. 20 (2009) 1.763-1.770.

[5] V. Amigó, M.D. Salvador, F. Romero, C. Solves y J.F. Moreno, J. Mater. Process. Tech. 141 (2003) 117-122.

[6] H.D. Kunze, Metal Powder Report 50 (1995) 36.

[7] G. Ryan, A. Pandit y D.P. Apatsidis, Biomaterials 27 (2006) 2.651-2.670.

[8] Z. Esen y S. Bor, Scripta Mater. 56 (2007) 341344.

[9] C. Aparicio, F. J. Gil, A. Padrós, C. Peraire y J. A. Planell, Rev. Metal. Madrid 34 (№. extra) (1998) 184-189.

[10] I.M. Robertson y G.B. Schaffer, Powder Metall. 53 (2010) 27-33.

[11] C.F. Li, Z.G. Zhu y T. Liu, Powder Metall. 48 (2005) 237-240.

[12] C.E. Wen, M. Mabuchi, Y. Yamada, K. Shimojima, Y. Chino y T. Asahina, Scripta Mater. 45 (2001) 1.147-1.153.

[13] M.V. Oliveira, A.C. Moreira, C.R. Appoloni, R.T. Lopes, L.C. Pereira y C.A.A. Cairo, Mater. Sci. Forum 530-531 (2006) 22-28.

[14] C.R.F. Azevedo, D. Rodrigues y F. Beneduce, J. Alloy. Compd. 353 (2003) 217-227.

[15] P.G. Esteban, L. Bolzoni, E.M. Ruiz-Navas y E. Gordo, Rev. Metal. Madrid 47 (2011) 169 . 187.
[16] V. Amigó, L. Reig, D.J. Busquets, J.L. Ortiz y J.A. Calero, Powder Metall. 54 (2011) 67-70.

[17] L. Reig, V. Amigó, D. Busquets y J.A. Calero, Powder Metall. 54 (2011) 389-392.

[18] M. Bram, H. Schiefer, D. Bogdanski, M. Köller, H.P. Buchkremer y D. Stöver, Metal Powder Report 61 (2006) 26-31.

[19] X. Zhao, H. Sun, L. Lan, J. Huang, H. Zhang y Y. Wang, Mater. Lett. 63 (2009) 2.402-2.404.

[20] R.M. German, Powder Metallurgy and Particulate Materials Processing, Metal Powder Industries Federation, New Jersey, USA, 2005, pp.121-260.

[21] C. Tojal, J. Devaud, V. Amigó y J.A. Calero, Rev. Metal. Madrid 46 (№ extra) (2010) 26-32.

[22] L.J. Gibson y M.F. Ashby, Cellular Solids: Structure and Properties, 2nd ed., Cambridge University Press, Cambridge, UK, 1997, pp. 175-231.

[23] R.M. German, Powder Metallurgy Science, 2nd ed., Metal Powder Industries Federation, New Jersey, USA, 1994, pp.241-299.

[24] R.M. German, G.L. Messing y R. G. Cornwall, Sintering Technology, Marcel Dekker inc., New York, USA, 1996, pp. 349-430.

[25] C. Leyens y M. Peters, Titanium and Titanium Alloys. Fundamentals and Applications, ed.Wiley VchGmbh\&Co., Weinheim, Alemania, 2003, pp. 423-424. 